\title{
Priority Setting in The Brazilian Emergency Medical Service: A Multi-Criteria Decision Analysis (MCDA)
}

Talita Dias Chagas Frazão ( $\nabla$ thalytachaggas@ufrn.edu.br)

Universidade Federal do Rio Grande do Norte https://orcid.org/0000-0003-2876-3168

Ana FA Santos

Universidade do Estado do Rio Grande do Norte

Deyse GG Camilo

Universidade Federal do Rio Grande do Norte

João FC Junior

Universidade do Estado do Rio Grande do Norte

Ricardo Pires Souza

Universidade Federal do Rio Grande do Norte

Research article

Keywords: Public health, Emergency medical service, Prioritizing victims, Prioritization criteria, Resources Shortage, Interactive and flexible method

Posted Date: October 1st, 2020

DOl: https://doi.org/10.21203/rs.3.rs-73633/v1

License: (c) (i) This work is licensed under a Creative Commons Attribution 4.0 International License.

Read Full License 


\title{
RESEARCH
}

\section{Priority setting in the Brazilian emergency medical service: a multi-criteria decision analysis (MCDA)}

\author{
Talita DC Frazão*, Ana FA Santos, Deyse GG Camilo, João FC Junior and Ricardo P Souza
}

\footnotetext{
*Correspondence:

thalytachaggas@ufrn.edu.br Departamento de Engenharia de Produção, Centro de Tecnologia Universidade Federal do Rio Grande do Norte, 59072-970, Natal, BR

Full list of author information is available at the end of the article

${ }^{\dagger}$ Equal contributor
}

\begin{abstract}
Background: Multicriteria Decision Analysis is a tool capable of supporting decisions with multiple criteria. Notwithstanding its confirmed value in the health area; so far, no studies have been found to help prioritize victims in the Emergency Medical Service, EMS. Since decision making within EMS involves multiple criteria, it is essential to find techniques and tools that encompass such elements, as to reduce errors. As to address this gap, the current research developed a multicriteria decision model to help prioritizing victims in the Brazilian EMS, which are still managed as a manual task.
\end{abstract}

Methods: To reach such endeavour, it was formed an expert panel and a discussion group, tasked to define the limits of the problem, and to identify the evaluation criteria for choosing a victim, amongst four alternatives derived from clinical and traumatic diseases scenarios of absolute priority in emergency situations occurrences. For prioritization, an additive mathematical method was utilized, aggregating criteria in a flexible and interactive version - FiTradeoff.

Results: The present work contributed to victims' prioritization by using the multicriteria decision support methodology which led to the identification of twenty-five evaluation criteria to guide the decision. It was noted that the protocols to guide regulating physicians do not consider all the criteria for prioritizing victims in an environment of resource scarcity. In the prioritization simulation composed of four demanding victims and only one available ambulance, the proposed model supported the decision by suggesting the prioritization of Victim 2.

Conclusions: From the identified improvement points, the developed decision model was able to improve the regulatory action of medical professionals. The elicitation procedure enabled the identification of criteria that, albeit well known, were not formalized by the current guidance protocols, which could contribute to contradictions and conflicts across the decision chain. Last, but not least, the proposed model could support decision making under the guarantee of a rational and transparent decision-making process that could be applied in other EMS.

Keywords: Public health; Emergency medical service; Prioritizing victims; Prioritization criteria; Resources Shortage; Interactive and flexible method

\section{Background}

The complexity of decisions increases whenever the issue of multiple criteria is present. However, errors can be reduced when the interest of the involved parties the preferences of the decision maker and all relevant criteria - are considered for 
the alternative of decisions analysis. Thus, if all the criteria as well as the different interested parties are recognized and inserted in the decision process, as to obtain the alternative analysis, the errors may be reduced. Faced with such scenarios, it is essential to look for techniques that include in the decision-making process, the greatest number of criteria as well as individuals involved. Multicriteria Decision Analysis, MCDA, is configured as a set of mechanisms with a structured and explicit approach to supporting decisions with multiple criteria when incorporating qualitative information, which in its turn, makes possible the improvement of the decision quality $[1,2]$.

Despite the proven value of the MCDA's support in the health care area, no models were found that used a multicriteria method to assist in prioritizing victims' decisions at Emergency Medical Services, EMS. This is one of the most important health services, as it plays a vital role in saving people's lives and reducing the rate of mortality and morbidity [3].

The use of MCDA as a method to support the establishment of health care priorities is not new. Some researchers show its growing importance in the health care area $[4,2,5,6]$, especially in the context of public health systems $[7,8,9,10]$. This is due to the combination of restricted resources and growing demands, which have led decision makers to address this issue more directly than in the past [11].

Notwithstanding the fact that the importance and sensitivity of decision-making in the field of EMS has been recognized by Operations Research scientists, emergency medical planners as well as health professionals who have studied strategic, tactical and operational problems for EMS since the 1960s.

Notwithstanding the fact that the importance and sensitivity of decision-making in the field of EMS has been recognized by operations research scientists, emergency medical planners as well as health professionals who have studied strategic, tactical and operational problems for EMS since the 1960s [12]. It was noted that what has been widely discussed are monitoring, forecasting and location problems [13, $14,15,16,17]$. Although studies by Carvalho et al. present generic approaches to optimize dispatch and relocation decisions and thereby maximize the preparation of the response system; they do not take into account fundamental criteria for the decision to dispatch ambulances, such as those related to the victim's severity and traffic conditions [18].

In Brazil, those problems are still addressed manually, with responsibility for the dispatch decision attributed to the regulating physicians of the Mobile Emergency Care Service (Serviço de Atendimento Móvel às Urgências - Samu/192), which is the mobile prehospital component of the Brazilian Urgency and Emergency Care System in municipalities and regions throughout the national territory.

The proposed model by the current work, aims to support the decision to prioritize victims attended by SAMU/192 regulating physicians and focuses on level 1 calls (red code of absolute priority), since they have the highest number of occurrences. Hence, throughout the text the term SAMU/192 will be used as a term equivalent to the Brazilian EMS.

The SAMU/192 strategy for ambulance sending follows protocols that are formulated based on criteria related to the victim's severity level. In the majority of cases, the victim who is closest to the available ambulance is attended firstly. However, in 
an environment in which the demand exceeds the capacity of the available resources, the greatest number of attributes related to the alternatives should be evaluated, because from a system overview, this decision can lead to better area coverage, considering not only the current situation, but also possible future emergencies.

The current lack of MCDA application to help prioritizing victims in EMS, and more specifically in SAMU/192 Brazil, denotes a gap in the literature that needs to be addressed. As a result of that, the current work developed a multicriteria decision model to assist regulating physicians; approaching the dispatch problem as a decision to prioritize victims, in an environment in which resources are scarce and the demand is growing, utilizing instruments such as a panel of experts, composed to structure the decision model. Finally, under the MCDA approach, the additive mathematical method in a flexible and interactive version was applied for the aggregation of criteria and subsequent prioritization of victims.

\subsection{Multicriteria Decision}

A multicriteria decision problem occurs when the Decision Maker, DM, an individual to whom power and responsibility over the decision are attributed - is faced with a situation with at least two alternatives for action, both of which are eligible for a solution, so that choice between the courses of action available is driven by the desire to meet multiple conflicting objectives [19, 20]. Although the responsibility for the decision is assigned to a well-identified DM, on many occasions the decisionmaking process is the product of the interaction between his preferences as well as other individuals who also influence the decision [21].

An important conceptual clarification is needed to understand the procedures related to MCDA: The term decision model refers to the simplified representation of the decision problem under analysis, belonging to a real system or situation; whereas the decision support method or MCDA method consists of the methodological theory or formulation, with a well-defined axiomatic structure, which can be used as an instrument for building a multicriteria decision model.

Rigorous concepts, formalized models, precise calculation procedures and axiomatic results must support the scientific analysis of the decision. However, under the isolated aspect of the mathematical model it is not possible to say that the decision is good or bad since the organizational, pedagogical and cultural elements that affect throughout the decision-making process must also be understood as factors that contribute to the quality and success of the decision [21]. The potential of MCDA in support of decision-making contexts is justified by the axiomatic structure described. Furthermore, evidence presented by Belton as to the limited capacity of the human being in the simultaneous consideration of multiple information argue in favour of the use of MCDA [22].

According to Keeney [23], the axioms that delineate Decision Analysis provides science with a structured and systematic methodological profile, as it follows from such logical principles that the attractiveness of alternatives must depend on the probabilities of occurrence of the possible consequences of each alternative and on the preferences of the decision-makers regarding consequences. The practical implication of the axioms is that the probabilities and utilities are capable of providing a theoretical and methodological basis that considers the judgments and values of the actors when evaluating the alternatives. 
Before selecting and implementing any method for MCDA, one must define the limits of the problem to be addressed, the more accurately the problem is defined, the more precise will be the analysis results [1]. The definition of the problem has happened, mainly, through discussion groups, formed by interdisciplinary teams and based on the knowledge and experience of the decision makers about the problem in question, with the possibility of consulting the relevant literature as well as specialists in the field [5].

Given that the problem was pre-defined, the objective clearly elaborated and the criteria to assist in the decision making well established, then a multicriteria evaluation method can be chosen to meet the conditions and specificities of the problem to be addressed [24]. Decision problems can be classified into six different types, for which MCDA can be useful: The problem of a) selection; b) classification; c) ordering; d) description; e) design; and e) the portfolio problem [21, 22].

\subsection{Multicriteria model of deterministic additive aggregation}

The term additive model will be used throughout the text as a reference to the MCDA model that uses a mathematical method of aggregating criteria with the characteristics that will be described below.

An additive model for aggregation uses a unique synthesis criterion type approach, which has as its main feature the aggregation of multiple criteria into a single synthesis [20]. In this model, uncertainty is considered in obtaining the vector of consequences $x$ for each alternative $a$.

For the problem of choice, which defines the class of problems in which the objective is to support the decision by choosing a subset of the action space, the solution of the additive model consists in the selection of the alternative that presents the greatest global value $v(a)[21]$. As the contribution of the additive model to the single criteria methods of synthesis lies in the process of aggregating the criteria,

in these methods the evaluation of the alternatives is carried out through the value function defined on the consequences, considering that each alternative is associated with a consequences vector $x[20]$.

Elicitation, the process of obtaining scale constants for each criterion is probably the main concern of an additive model regarding the aggregation of value functions $v i(x i)$ over the consequences $x i$ for all criteria $i(i=1, \ldots, n)$. This is expressed below in Equation 1:

$$
v(x)=\sum_{i=1}^{n} k_{i} v_{i}\left(x_{i}\right)
$$

The greatest challenge faced by MCDA lies in the assessment and modelling of preferences [21]. However, preferences can be modelled by rules and logical relationships [25]. There are four elementary binary relationships capable of addressing the decision maker's preferences: (i) $a \mathrm{I} b, a$ is indifferent to $b$; (ii) $a \mathrm{P} b$, a is strictly preferred to b; (iii) $a \mathrm{Q} b$, it is the hesitation between indifference and preference; (iv) $a \mathrm{R} b$, the hesitation is between $a \mathrm{P} b$ and $b \mathrm{P} a[26,27]$.

What methodologically distinguishes MCDA methods from one another is how the process of aggregating the binary relationships is performed. For methods based on 
the additive model, aggregation is established by the unique synthesis function, which addresses and represents the preferences of the decision maker. This function is useful for evaluations and comparisons between alternatives to be carried out and, given the theoretical and axiomatic reality of these methods, it is assumed that the decision maker's preference structure is $\mathrm{P}, \mathrm{I}$, through which it is possible to solve the problem. based on the additive model [27].

Knowing the preferences of the decision maker, a problem can be solved based on the additive model, for that it is still necessary two types of evaluation: the intracriteria and the intercriteria. The intracriteria assessment aims to evaluate each alternative $i$ for each criterion $j$, which lead to the value function $v_{j}\left(a_{i}\right)$, the construction of the value function for each element is based on the evaluation of the consequences to be obtained. In the intercriteria evaluation with the information $v_{j}\left(a_{i}\right)$, what we are looking for now is the information that considers the combination of the different criteria, and for that, it is necessary to choose a method of criteria aggregation [20]. When the criteria are represented by different units, it is necessary to normalize these values, so that they are redefined on a scale from 0 to 1.

Prior to the final recommendation, sensitivity analyses can be carried out to investigate whether the preliminary conclusions are robust or whether they are sensitive to changes in aspects of the model. Based on the results, changes can be made to investigate the meaning of the lack of information, to explore the effect of a decision maker's uncertainty on his values and priorities, or to offer a different perspective on the problem [22].

\subsection{FiTradeoff}

Almeida et al. proposed the Flexible and Interactive Tradeoff method, FITradeoff, to meet the problem of eliciting scale constants, which will occasionally be referenced also by weight of the criteria [28]. In the FITradeoff the dimensioning of the scale constants is based on the procedure of tradeoff in which it is intended to achieve the tradeoff of values, defined by the moment when the decision-maker is indifferent to two consequences and can be willing to exchange them [28, 20]. Such procedure is adopted in the Tradeoff method, what makes FITradeoff to be considered its extension $[23,29]$.

The concept of flexible elicitation is one of the positive aspects of the abovementioned method because it improves the elicitation procedure by tradeoff. The flexibility attributed to FITradeoff means that the process of dimensioning the scale constants can be easily changed and adapted to different conditions and circumstances, allowing the weights to be revised as adversities observed during the elicitation are noticed. The method is set in a Decision Support System (DSS).

In compensatory methods like this, the DM considers the compensations by criterion when comparing the consequences of the alternatives [23, 29]. Therefore, the decision maker is invited to choose between two key consequences, both with the best performance in one of the criteria $\left(b_{i}\right)$ the worst performance $\left(w_{i}\right)$ in all other criteria. The operationalization through the DSS includes the following steps: Step 1. Intracriteria assessment; Step 2. Classification of criteria weights; Step 2.1. Attempt to solve the problem using the set of weights available; Step 3. The preferences of the DM are evaluated in order to arrive at the results. 
The first part allows the weight spaces to be defined. After this step, the second part is started, difference can be seen between the procedure and the traditional model, the DM is not required to define an exact value of $\left(x_{l}^{i}\right)$, which represents the criteria $i$ result, for which the indifference is obtained between the consequences, whilst in the traditional method such value is required.

As the second part begins, a new weight space can be obtained, which is a subspace of the previous step, in which all valid relations for the next step are considered. If the alternative is identified, the DSS classifies the alternatives in three situations: potentially ideal, dominated or optimized. If the solution is not found, then the third stage begins, that is, that of assessing the preferences of the DM that can be divided into four stages: Stage 1. Define values to test the weight distribution; Stage 2. Request the DM to indicate their preferences; Stage 3. LPP computing; and Stage 4. Finalization.

Thus, the objective is to find an alternative, based on the vector of the alternatives, which has the maximum value according to the weight of the criteria space [30]. Therefore, PPL is performed until an ideal alternative is found. If not, the dominated alternatives are eliminated and the process is started again, from the stage 1. Subsequently, only the alternatives identified as potentially optimal are considered in the subsequent steps, otherwise the process is finalized. [28]. In the finalization step, the weight ranges supporting the solution are computed and produced in a report with the final recommendation.

\section{Methods}

\subsection{Study location and decision-making actors}

The Northeast Region of the Brazilian territory is composed of nine states: Alagoas, Bahia, Ceará, Maranhão, Paraíba, Pernambuco, Piauí, Rio Grande do Norte and Sergipe. Its territorial extension is 1,554,257.0 square kilometres, being the third largest regional complex in Brazil. According to data from the 2010 Demographic Census, carried out by the Brazilian Institute of Geography and Statistics (IBGE), the North-eastern population totals 53,081,950 inhabitants, housing about $28 \%$ of the resident population in Brazil.

The current research is based on the specialized knowledge of representatives of the nine states of the Northeast Region of Brazil. An expert panel was formed by fourteen medical regulators who contributed to the structuring of the decision problem through semi-structured questionnaires. Carrying out the structuring phase with a diverse group of stakeholders enriches the process, as it provides a common view of the problem and allows the integration of different perspectives [31].

The face-to-face observations and discussions took place at SAMU/192 - Natal in Rio Grande do Norte, RN, with a discussion group formed by the General Director, the Medical Coordinator, the Regulation Coordinator and one of the most experienced on-call heads at the regulation centre. Everyone could express their preferences regarding the cases presented. The decision maker chosen was the General Director of SAMU/192 - Natal, who is also a regulatory physician. An analyst mediated the decision-making process, it was not necessary to use techniques to reach a consensus amongst group members. Other stakeholders were selected based on simultaneous participation throughout the study. 
The participation of those Stakeholders endeavoured to support the proposal and development of a transparent and rational decision model, which could maximize the benefits as well as minimizing the risks in the Samu/192 Brazil environment, as reproducibility and validation are two important parameters that need to be considered when dealing with the challenge of the decisions of regulating physicians. Bearing in mind that learning, experience, literature, inductive and deductive reasoning, the correct interpretation of signs and symptoms, the gathering of evidence for decision making, are all necessary items for medical judgment and there is a great deal of difficulty in reproducibility and validation of these items [32].

\subsection{General aspects of medical regulators decision}

During the development of the multicriteria decision model, the entire path from the call to the transport of the victim to a health unit was analysed, as no information important to the decision, which occurs in real time, could be disregarded.

Assistance to victims starts from the telephone call, when guidance is provided on the first actions. The call is answered by medical regulation technicians - TARMs who identify the emergency and collect the first information about the victims and their location Brasil2014.

After the reception and identification of the calls, requests are judged by the Regulatory Physician, RP, who classifies the level of urgency of each one, which defines the necessary resource for their adequate care, which can involve from a simple medical advice to the activation of a Radio Operator, RO, who will activate the nearest or appropriate ambulance, depending on the severity of the situation. As in Figure 1.

The operation of SAMU/192 is shown in Figure 1.

After the on-site assessment, the patient must be transported safely to those health services that can best serve each patient, responding resolutely to their needs and ensuring the continuity of care initially provided by SAMU. Due to its performance, SAMU constitutes an important link between the different levels of the Health Care System [33].

In the present article, it is sought to maximize the efficiency of the system by analysing the process of sending ambulances, using relevant information for decision makers, thus, it is aimed to prioritize victims with the available resources. Therefore, the decision context is analysed, including in the model the definition of important decision concerns and the items relevant to its evaluation on the identified criteria. This research follows three main phases: a) the decision problem is structured; b) the multicriteria method is selected; c) the preference modelling is performed.

\subsection{Structuring the decision problem}

2.3.1 Problem, objective and alternatives of the MCDA model

Two meetings with a discussion group were held at the Samu/192-Natal Regulation Centre between April and October 2018; resulting in: a) the initiation of the decision problem delimitation; b) the definition of the specialist panel; and c) the establishment of alternatives as four victims and for illustration four cases (two clinic and two traumas) were selected, all classified as Level 1, red code. The choice of topics was based on the SAMU/192 service history. When analysing a sample of 
historical data from 2015 to 2018, chosen for convenience, the results revealed the clinical and trauma cases as the most representative specialties, because together they account for $84.77 \%$ of the total number of diagnoses. The cases were elaborated based on the literature and the experience of the regulating physicians at the Natal-RN Regulation Centre [34].

\subsubsection{Rating criteria}

To identify the evaluation criteria, a mainly qualitative approach was chosen as being more appropriate to the exploratory nature of the study and to address the research question. In order to maximize the credibility, reliability and degree of confirmation of the findings, the qualitative research instruments that were employed in this phase included observations, document review, open and semi-structured interviews. The combination of such instruments enabled the most complete and multifaceted examination [35].

Observations and open interviews This stage of the study involved observing the field that did not participate in the daily work practices of professionals at the SAMU/192, Natal-RN, regulation centre, with a greater focus on Regulating Physicians, RPs. The objective of this initial stage of data collection was to have an initial understanding of the organizational context and the decision making of the $\mathrm{RP}$ in practice.

The observations also involved conducting open interviews with a number of key Regulatory Central employees $(\mathrm{n}=30)$ with potential profiles to provide an overview of the organization and service delivery (between December 2018 and June 2019). Such individuals included coordinators and medical regulators, whose roles alternated between operational and leadership management. Although the character of the observations was non-participant, the open interviews had the objective of capturing possible internal and external perspectives, on regulation and systemic influences. Observations were divided into a six-month time span both to reduce the observer's tendency to become active, avoiding interference and to avoid overloading new information to the point that subsequent data does not make sense [36].

Throughout the open interviews it as collected: a) demographic information, b) the relevant documentation dealing with policies and procedures; and c) the experience of the context of the decision to prioritize victims seen by the RPs. This allowed an understanding of the geographic area served, the population, the service provided, and the relevant national and local policies within the context. The data collected in the observations and open interviews were part of the necessary subsidy, for the elaboration of the semi-structured interview, enabling the identification of twenty-one decision criteria that were categorized in five dimensions related to a) the health system; b) the support; c) the victim; d) the victim's health status; and e) external factors.

Document review The protocols for advanced and basic life support used within the organizational environment were analysed alongside to the criteria preestablished by the support system of the regulation centre, the latter being used 
for the investigation of the presumed severity, during the call. With the analysis of such documents, four of the six criteria related to the victim's health were extracted $[37,38]$.

At the end of this stage, amongst the data collected, there were twenty-five decision criteria for the problem of prioritizing victims, subdivided into five dimensions. These data were presented to the discussion group, in a meeting, in which it was decided that these criteria would undergo a review and a second validation. This time, it would be carried out by the regulating physicians from other north-eastern states.

Semi-structured interviews The final step in selecting the criteria involved conducting semi-structured interviews with SAMU/192 representatives from all northeastern states. An online questionnaire was developed based on data collected from observations, open interviews and document reviews. A total of fourteen regulating physicians participated; amongst them eleven were in leadership positions and had between five and ten years of experience in medical emergency.

With regard to the questionnaire, the presentation of the questions was preceded by the presentation of the central problem to the participants as a scenario in which doctors receive three calls with the same characteristics, three victims who complain of pain, and to answer them they have only one available ambulance. There was also the observation that cases should be treated as an urgent priority. After this introduction, two questions were presented to the physicians. The first question asked: What do you need to know to make the decision? What can influence this decision, knowing that the protocol advises the dispatch, and yet resources are scarce? Check the options you need to make this decision, check as many as needed. The dimensions and their respective criteria were presented, and the doctors could mark as many as they thought necessary. The second question investigated: At the time of the decision, based on your experience and skills, could any other criterion or dimension not present in the protocols or in this Questionnaire be added?

\subsection{Selecting a multicriteria method}

For selection of the multicriteria method, basic preference situations were observed to support the decision maker's preference modelling, as well as the rationality considered by the DM to the context under study [27]. With the definition of the list of criteria and their corresponding scale constants, the intracriteria assessment was performed, obtaining the value functions $v_{i}\left(x_{i}\right)$ for each criterion $i$ which, in its turn, enabled the construction of the decision matrix.

The decision maker's preference was considered linear for all criteria [39]. For the standardization procedure applied in this work, the scale is from 0 to 1 , in which 0 and 1 represent, respectively, the minimum and maximum values of the performances in the criterion. The standardization procedure is necessary since the form of partial information used and the weight limits are obtained indirectly according to the tradeof [28].

\subsection{Modelling preferences}

With the discussion group, the elicitation of the scale constants - the intercritical evaluation - was carried out with the FITradeoff multicriteria method, in the 
software FITRADEOFF for the additive model of choice with sensitivity analysis, (code FU-T1EEMO-CT1).

At this stage, one of the criteria was chosen to be used in the evaluation so that this choice was based on the idea of which would require less cognitive effort from the decision maker to perform the comparisons. The DM was asked, through the DSS, to choose one of the criteria. The decision maker was instructed to consider some issues related to the ability to interpret each result. In this step, the analyst supported the task. With the support of the DSS, there was an attempt to resolve through the available weight space, so that the interaction was finalized when a single solution was found. In the finalization step, the solution weight ranges were computed and a report was produced with the final recommendation.

Sensitivity analyses were also performed with all the criteria in order to examine the robustness of the selection and identify criteria potentially sensitive to weights. The percentage values for variation of the criteria were $-20 \%$ and $+20 \%$. The consequence value of each alternative $j$ from the problem to the criterion $i$ assumed a value $S j, i$ such that $(p j, i) *(0,9) \leq S j, i \leq(p j, i) *(1,1)$ in which $p j, i$ corresponds to its nominal value (value of the original consequence matrix). To maintain the original limits of the consequence space, the DSS verifies the minimum value of the consequence space generated in the Sensitivity Analysis cycle and assigns the minimum nominal value to it. The same is done for the maximum nominal value.

\section{Results}

\subsection{Structuring the decision problem}

\subsubsection{Problem, objective and the MCDA model alternatives}

Based on the objective of prioritizing victims, the main issue is the choice of which victim to attend first. To this end, the alternatives presented were illustrated based on four occurrence scenarios that brought physicians the main points of pre-hospital care in emergency situations and clinical and traumatic emergencies, these classified as emergencies of absolute priority. The Table 1 presents additional information about the four cases presented to the professionals.

Additional information on the four cases presented can be seen on Table 1.

Victim 1 Call answered by the Assistant Medical Regulation Technician (TARM) from 192. Complaint: "tightness in the chest and difficulty breathing". The applicant for the Mobile Emergency Care Service (SAMU/192), the victim's co-worker, told TARM that a 22-year-old military firefighter was attacked by a swarm of African bees. The accident occurred when he was carrying out a training exercise in a region far from the municipality. At the time of the request, the victim had hives, itching of the body and chest discomfort associated with sudden onset severe dyspnoea, at the time of the occurrence it started raining on the spot, the applicant insistently warned the Regulating Physician that the victim was allergic to bee stings. It was authorized by the doctor to the Advanced Support Unit (USA).

Diagnostic Hypothesis: anaphylaxis / poisoning syndrome.

Victim 2 Call answered by the Assistant Medical Regulation Technician (TARM) at 192. Requested Cause: motorcycle-bicycle collision. A passerby activates the 
Mobile Emergency Service (SAMU/192) reporting to TARM that he witnessed a motorcycle-bicycle collision, leaving a male victim, apparently aged 40, who was unconscious, breathing, and bleeding profusely. To the regulating physician, the applicant reported that the victim was riding the motorcycle without a helmet and that, at the moment, he was not responsive and had severe bleeding from the nose and ear. The Advanced Support Unit has been authorized.

Diagnostic Hypothesis: severe traumatic brain injury.

Victim 3 Call answered by the Assistant Medical Regulation Technician (TARM) at 192. Cause requested: motorcycle fall. Requestor activates Mobile Emergency Service (SAMU/192) to assist a 23-year-old victim of a motorcycle fall, who was conscious, breathing and bleeding profusely. To the regulator, he reported that the victim was driving the vehicle without wearing a helmet, lost consciousness at the time of the accident and had a severe head injury. It was sent to the Basic Support Unit for the location. During treatment, the patient evolved with an episode of post-traumatic tonic-clonic seizure crisis. The Regulating Physician has authorized the Advanced Support Unit for the Site.

Diagnostic Hypothesis: moderate traumatic brain injury, with evolution of tonicclonic post-traumatic seizure crisis.

Victim 4 Call answered by the Assistant Medical Regulation Technician (TARM) at 192. Cause requested: Difficulty breathing. The applicant, a relative of the victim, calls the Mobile Emergency Service (SAMU/192), and informs the TARM that a 60 -year-old was at home when he was sick with difficult breathing. He reported to the regulating physician that the victim was hypertensive, had heart disease and had sudden dyspnoea followed by syncope and decreased level of consciousness. The applicant also found that the pulse was palpable, but very weak.

Diagnostic Hypothesis: Respiratory insufficiency, plus a decreased level of consciousness.

\subsubsection{Rating criteria}

Based on the analysis of the decision problem faced, it was concluded that although the criteria that guide regulatory physicians are known, there were other criteria that influenced decision making, but that were not provided for in the protocols. Since prioritizing victims is a complex decision, with many determinants, it was necessary to investigate what might influence this decision, knowing that the protocols that regulate the decision to send ambulances advise immediate assistance, but nevertheless, resources are scarce. Faced with such a scenario, which victim should be prioritized?

Therefore, in this phase for structuring the decision problem, the criteria that guide the evaluation of alternatives were identified. Thus, after the period of observations, open interviews and document review, it was concluded that the information investigated by the regulating physicians with the potential to influence at the time of the call may be of the following distinct types:

1 Criteria related to the Health System: access to the doctor, access to the bed, support from security agencies; 
2 Criteria related to Support Tools: ambulance location, access to the assistance system, access to support material;

3 Criteria related to the Victim: location of the victim, proper means of transportation, age, time of call, health insurance, sex, refusal, desire;

4 Criteria related to the Victim's Health Status: airway and oxygenation, state of consciousness, pulse, traumatization, victim's health history, alcohol or drug intake;

5 Criteria related to External Factors: road traffic congestion; social commotion; bad weather, hard to reach address, insistence of people close to the victim .

The semi-structured interviews - prepared based on the categories of criteria identified, aimed at selecting the important criteria for prioritizing victims, classified as absolute priority, in a situation of scarcity of resources - were sent to the panel of experts. The results are shown in Figure 2, wherein it can be seen that the three main ones are related to the victim's health status, followed by access criteria and the victim's location - investigating whether the victim is able to access the doctor seems to be decisive. Desire, refusal, sex, health insurance and access to the bed, in most cases, are not priority information for specialists. The other criteria can be considered important in a decision to prioritize victims as the doctors have shown interest.

Decision criteria evaluated by the group of experts presented in Figure 2.

The second question investigated which other criteria or dimension not met in the protocols or in the questionnaire itself could be added. According to the answers presented, the experts agreed with the classes of criteria presented and did not add any other to the list. This result was presented to the discussion group.

\subsubsection{Selection of evaluation criteria}

Several dimensions of performance can be considered when estimating the set of victim prioritization criteria. These criteria may include the location of the ambulance, access to the doctor, the age and state of consciousness of the victim, as well as whether the case is characterized by social commotion. However, it is not possible to determine a universal set of victim assessment criteria in a single model because each case has specific performance criteria. For the cases under evaluation, the discussion group agreed that the list would be complete if it contemplated the ten criteria described in the Tables 2 and 3.

The final list of criteria related to the victim's health is shown in Table 2.

Access to the service system and support material was considered to exist and would not influence the decision. Regarding the time of the requests, it was preestablished that the calls were registered in the same time interval. Finally, with regard to desire and refusal, it was considered that both the family and the victim wanted care. The location of the ambulance, the intake of alcohol or drugs, the support of the security agencies, access to the bed and the sex of the victim were not considered for the evaluation of the four applicants since the discussion group would not be relevant criteria for these cases.

The final list of criteria related to the victim and external factors is shown in Table 3 . 
The other criteria were included in the model and in order to facilitate and streamline the decision-making process, those that had similarities were grouped: the assessment of access to the victim's location took into account the access difficulties motivated by both roads and vehicles (traffic congestion) road + difficult to reach address), the social commotion investigated information about popular indignation and the climatic conditions to which the victims were exposed and, finally, regarding the criterion of access to the doctor, the regulating physician evaluated if the victim had any condition that would allow him have access to the doctor safely (health plan + proper means of transportation). Among the ten selected criteria, eight are on a verbal scale ranging from 1 to 4 .

The objective, criteria and alternatives of the proposed model are presented in Figure 3.

The Figure 3 presents the summary of the results found so far: the objective of the decision model, the ten criteria and the alternatives.

\subsection{Selecting a multicriteria method}

The decision-making situation was considered as a whole and the theoretical and technical characteristics of the multicriteria FITradeoff method were analysed for the problem of choice, which were compared to the information already structured as to justify the method choice.

It was observed that the input information is in accordance with the method under analysis since the DSS operates with numeric inputs. In addition, the decision-maker presented compensatory rationality, in which it is assumed that there may be an absolute compensation between the different assessments, given that the reality of the structured decision problem, a good performance in 'airways and oxygenation' can easily offset a poor performance in 'victim's history'. This rationality profile is in line with that for the application of FITradeoff.

In order to comply with the aggregation procedure appropriate to the axiomatic structure of FITrdeoff, performance aggregation was carried out based on views of tradeoffs between various consequences, realized through comparisons presented to the decision maker. As a result of that, a unique synthesis function was described, an element that represents the decision maker's preference, having been chosen because it is useful for evaluations and comparisons between alternatives. The overall performance leads to a total pre-order of the model's alternatives.

Since the decision maker was able to make comparisons between pairs of consequences with strict preference and indifference relations, the preference structure $(\mathrm{P}, \mathrm{I})$ was sufficiently adequate for modelling the decision maker's preferences. As the approach of the single synthesis criteria methods does not accept that there may be good reasons to justify the incomparability between two alternatives, in this situation it was admitted that the alternatives can be compared with each other, therefore, there is no presence of incomparability. The transitivity property was also observed.

The decision support system (DSS) that accompanies FITradeoff is an important aspect, as it was built under a flexible elicitation concept that requires less cognitive effort from the decision maker because it requires reduced information, without the need for adjustments of indifference between the consequences. This makes it 
easier for the decision maker to compare results based on strict preference and not indifference [28].

\subsection{Modelling preferences}

The table 4 evaluates each alternative $i$ for each criterion $j$, which leads to a value function $v_{j}\left(a_{i}\right)$, corresponding to intracriteria evaluation. The table shows the format of the input data of the chosen multicriteria method. Information about the classification of the criteria (whether continuous or discrete) must be added which is the type of function that each criterion will assume. In the case of discrete criteria, it is necessary to inform the number of scale levels to be considered. This information is presented in the next section.

The decision matrix is shown in Table 4.

After structuring the problem considering an additive model, the data in Table 4 served as input to the DSS. Then, the FITradeoff steps were operationalized in a meeting with the discussion group, after the ordering of the criteria, flexible elicitation started, two questions to the decision maker were sufficient for a single solution found for the weight space. The recommendation was to prioritize Victim 2. Figure 4 illustrates the DSS output, presenting the space of viable weights for which Victim 2 is suggested. The breadth of the range of values demonstrates the robustness of the result in view of the variety of weights associated with the main criteria, in decreasing order, for which Victim 2 would still be suggested.

Weight ranges are shown in Figure 4.

The weight range for each criterion is: Airways and oxygenation $(0.75-0.1)$; State of consciousness (0.5 - 0.08); Pulse (0.33 - 0); Traumatization (0.25 - 0); Victim's health history $(0.2-0)$; Access to the doctor (0.17 - 0); Access to the victim's location (0.14 - 0); Social commotion (0.13 - 0); Location (0.11 - 0); and Age $(0.1-0)$. These weight ranges result from LPP models, considering maximum and minimum weights, subject to restrictions for the development alternative.

\subsubsection{Análise sensibilidade}

In the sensitivity analysis, executed with all the criteria to examine the robustness of the selection and to identify criteria potentially sensitive to weight, the percentage values for the variation of the criteria were $-20 \%$ and $+20 \%$.

The alternatives found during the performance of the sensitivity analysis are shown in Figure 5.

The Figure 5, illustrates a graph with the alternatives that were present in the subset of potentially optimal alternatives during each cycle of Sensitivity Analysis. Among these alternatives, those that were also present in the original subset appear in blue. Those that were present in the subset in some Sensitivity Analysis cycle but were not in the nominal subset are shown in purple. The impact of changes in the selected alternative is also observed, which suggests that Victim 2 remains consistently the most attractive alternative, whatever the assumed value.

\section{Discussion}

The approach to the regulation process of emergency services from the perspective of the MCDA methodology made it possible to formalize the decision-making process 
carried out by regulating physicians and others players at SAMU/192. The analysed decision context has as main objective the prioritization of victims in a scenario of scarcity of resources. With the application of FITradeoff, a method appropriate to the specificities of the problem, the decision maker's preferences modelling and intracriterial evaluation were performed. The parameterization of the method, carried out in the intercritical evaluation stage, observed the structure defined by the additive model, in which the evaluations of the alternatives are aggregated according to the computed value functions and constants of elicited scales, having been the elicitation procedure performed by FITradeoff.

However, before the prioritization of alternatives was finalized, it was necessary to revisit the structuring phase of the decision problem, regarding the identified criteria. This occurred given that the first suggestion of prioritization was not validated by the discussion group; hence, the analyst-actor interaction was essential. Therefore, the questioning made by the professionals motivated the investigation of the discrepancy between what the model suggested and what would be performed by doctors.

The research conducted motivated the feedback of the methodological flow so that the model could be perfected and become credible to the real system, that is, in the context of the decision-making process for prioritizing victims of SAMU/192. It was perceived the need for some criterion that contemplated whether the victim was within reach or had access to medical care. Hence, the model that was previously formalized with nine criteria, had the inclusion of the criterion "Access to doctor" the MCDA process was then re-executed. The modelling of preferences and tradeoffs was conducted according to the new configuration of the decision problem, since the inclusion of a new criterion characterizes no longer the same object of analysis previously addressed. Finally, the modelling of preferences performed and Victim 2 was suggested to support the decision to prioritize by the model, which converged on the consensus reached by the regulating physicians to the hypothetical scenario described.

It is relevant to point out to the positive character of the methodology used due to the possibility of revising the previous steps before making the recommendation to the decision maker. It is important to bear in mind that the purpose of the MCDA is to support the decision, and not to impose the suggestion on the group.

Therefore, the flexibility of the MCDA process as well as the importance of the stage of structuring the problem are emphasized, considering its impact on the successor stages and its critical character regarding the application of the chosen method. In many cases, the richness of the multicriteria approach is due precisely to the discussions and a greater degree of understanding of the problem; as the structuring phase of the problem presented to all of those involved, criteria unknown to the protocols that regulate decisions, but still considered important for regulating physicians.

It was also observed that the majority of the evaluation criteria are common to all cases, although they are adapted to the special characteristics of different priorities (for example, the meaning of access to the doctor may differ if the criterion refers to the health system and not the victim). This common set of criteria allows the development of a uniform evaluation structure and the implementation of additional 
comparison analyses. However, there are some criteria that differ according to each case. For instance, the support attribute of security agencies is a priority in cases of psychiatric emergency. The contribution of Multicriteria Decision Analysis lies in the potential of the tool to formalize the decision process under study and not only to rationalize it, granting a degree of transparency that is increasingly required in the decisions of the public health system, due to the characteristic of demand exceeding the available service capacity.

\section{Conclusions}

Based on the definitions, propositions and theorem presented, it was possible to develop a multicriteria decision model to help prioritize victims, within the Mobile Emergency Care Service - Samu/192. Health care decision-makers require tools that consider a variety of complex factors related to multi-objective missions. Given such complexity, the decision to prioritize victims in an environment of scarcity of resources should allow even conflicting criteria to be taken into account in order to better support decision makers.

After structuring the problem, it was possible to learn about the twenty-five evaluation relevant criteria. However, it was concluded that for the decision prioritization on the four cases presented; only ten of those criteria related to the victim's state of health, the victim itself and external factors would be relevant. However, that conclusion does not invalidate the other criteria, as they may be necessary in different contexts of prioritization. For instance, in a psychiatric case, the criterion "support from security agencies" will be part of the decision. It is important to highlight the importance of the criterion "Access to the doctor", who, despite not being present in the protocols, is able to modify the model's result when not considered.

It was also noticed that the decision-maker manages to identify relations of preference and indifference between the pairs of consequences. In this situation of decision making, it was found that the alternatives can be compared with one another, which means that there is no presence of incomparability.

The transitivity property was also confirmed. The flexible and interactive Tradeoff process proved to be effective for prioritizing victims, within this context, since the model and the best decision presented were characterized as a simplification of reality, given the acceptance of the results by the participating doctors. Therefore, reproducibility and validation could be performed.

The selection and weighting of the criteria indicated that the protocols for guiding the prioritization of victims in an environment of scarcity of resources currently in force at SAMU/192 do not address all criteria considered by the regulating physicians since the factors subjective to the professional's decision had not been elicited in line with the reality of the decision-making process for prioritizing victims in emergency services, having been properly identified with the support of the MCDA methodology applied by this study.

The model developed has the potential to support the regulating physicians at SAMU/192 to direct resources to victims who greatest need of support, and can be used in other pre-hospital emergency care units. Hence, it is evident that there is a vast field of research exploration for Multicriteria Decision Analysis in prioritizing victims of pre-hospital emergency care, with the capacity to generate considerable improvements in the sector. 
Declarations

Ethics approval and consent to participate

Not applicable.

Consent to publish

Not applicable.

Availability of data and materials

Not applicable.

Competing interests

The authors declare that they have no competing interests.

Funding

Not applicable.

Author's contributions

TDCF and RPS conceived the study. TDCF, RPS and AFAS wrote and edited the manuscript. TDCF and RPS participated in data collection and analysis. TDCF, AFAS and RPS participated in interpretation of results. TDCF, JFCJ and DGGC participated in the review and translation of the text. All authors read and approved the final manuscript.

Acknowledgements

Not applicable.

Authors' Information

Not applicable.

\section{References}

1. Diaby, V., Goeree, R.: How to use multi-criteria decision analysis methods for reimbursement decision-making in healthcare: a step-by-step guide. Expert Review of Pharmacoeconomics \& Outcomes Research 14(1), 81-99 (2014). doi:10.1586/14737167.2014.859525

2. Thokala, P., Devlin, N., Marsh, K., Baltussen, R., Boysen, M., Kalo, Z., Longrenn, T., Mussen, F., Peacock, S., Watkins, J., ljzerman, M.: Multiple criteria decision analysis for health care decision making - An introduction: Report 1 of the ISPOR MCDA Emerging Good Practices Task Force. Value in Health 19(1), 1-13 (2016). doi:10.1016/j.jval.2015.12.003

3. OTONI, T.: Regimento Interno - Jequitinhonha (2012)

4. Marsh, K., Lanitis, T., Neasham, D., Orfanos, P., Caro, J.: Assessing the Value of Healthcare Interventions Using Multi-Criteria Decision Analysis : A Review of the Literature, 345-365 (2014). doi:10.1007/s40273-014-0135-0

5. Frazão, T.D.C., Camilo, D.G.G., Cabral, E.L.S., Souza, R.P.: Multicriteria decision analysis (MCDA) in health care: a systematic review of the main characteristics and methodological steps 1, 1-16 (2018). doi:10.1186/s12911-018-0663-1

6. Moreno-Calderón, A., Tong, T.S. \& Thokala, P.: Multi-criteria Decision Analysis Software in Healthcare Priority Setting: A Systematic Review. PharmacoEconomics 38 (2020). doi:10.1007/s40273-019-00863-9

7. Mühlbacher, A.C., Kaczynski, A.: Making Good Decisions in Healthcare with Multi-Criteria Decision Analysis: The Use, Current Research and Future Development of MCDA. Applied Health Economics and Health Policy 14(1), 29-40 (2016). doi:10.1007/s40258-015-0203-4

8. András Inotai, Huong Thanh Nguyen, Budi Hidayat, Talgat Nurgozhin, Pham Huy Tuan Kiet, Jonathan D. Campbell, Bertalan Németh, Nikos Maniadakis, Diana Brixner, K.W.\&.Z.K.: Guidance toward the implementation of multicriteria decision analysis framework in developing countries. Expert Review of Pharmacoeconomics \& Outcomes Research 18:6(585-592) (2018). doi:10.1080/14737167.2018.1508345

9. Chen, T.: A novel VIKOR method with an application to multiple criteria decision analysis for hospital-based post-acute care within a highly complex uncertain environment. Neural Comput \& Applic 31(3969-3999) (2019). doi:10.1007/s00521-017-3326-8

10. Camilo, D.G.G., de Souza, R.P., Frazão, T.D.C.e.a.: Multi-criteria analysis in the health area: selection of the most appropriate triage system for the emergency care units in natal. BMC Med Inform Decis Mak 38 (2020). doi:10.1186/s12911-020-1054-y

11. Ham, C.: Priority setting in health care: Learning from international experience. Health Policy 42(1), 49-66 (1997). doi:10.1016/S0168-8510(97)00054-7

12. Aringhieri, R., Bruni, M.E., Khodaparasti, S., van Essen, J.T.: Emergency medical services and beyond: Addressing new challenges through a wide literature review. Computers and Operations Research 78(August 2016), 349-368 (2017). doi:10.1016/j.cor.2016.09.016

13. Aringhieri, R., Bruni, M.E., Khodaparasti, S., van Essen, J.T.: Emergency medical services and beyond: Addressing new challenges through a wide literature review. Computers and Operations Research 78, 349-368 (2017). doi:10.1016/j.cor.2016.09.016

14. M. Reuter-Oppermann, P.L. van den Berg, J.L.V.: Logistics for emergency medical service systems. Health Syst. 6, 187-208 (2017) 
15. Bélanger, V., Ruiz, A., Soriano, P.: Recent optimization models and trends in location, relocation, and dispatching of emergency medical vehicles. European Journal of Operational Research 272(1), 1-23 (2019). doi:10.1016/j.ejor.2018.02.055

16. Reuter-Oppermann, M., Richards, D.: Decision support for EMS policy making using data analytics and real-time alerts. Proceedings - 2019 IEEE World Congress on Services, SERVICES 2019, 266-271 (2019) doi:10.1109/SERVICES.2019.00079

17. Andersson, H., Granberg, T.A., Christiansen, M., Aartun, E.S., Leknes, H.: Using optimization to provide decision support for strategic emergency medical service planning - Three case studies. International Journal of Medical Informatics 133(July 2019), 103975 (2020). doi:10.1016/j.ijmedinf.2019.103975

18. Carvalho, A.S., Captivo, M.E., Marques, I.: Integrating the ambulance dispatching and relocation problems to maximize system's preparedness. European Journal of Operational Research 283(3), 1064-1080 (2020) doi:10.1016/j.ejor.2019.11.056

19. Keeney, R.L., Raiffa, H.: Decisions with Multiple Objectives: Preferences and Value Tradeoffs. Cambridge: Cambridge University Press, United Kingdom (1993)

20. Almeida, A.D.T.: Processo de Decisão Nas Organizações: Construindo Modelos de Decisão Multicritério, São Pulo: Atlas (2013)

21. Roy, B.: Multicriteria Methodology for Decision Aiding, p. 303 (1996)

22. Belton, V., Stewart, T.: Multiple Criteria Decision: An I Ntegrated Approach. Springer, US (2002)

23. Keeney, R.L.: Value-focused Thinking: A Path to Creative Decision Making, London, England: Harvard University Press Cambridge, Massachusetts (1992)

24. Dolan, J.: Multi-Criteria clinical decision support 3, 229-248 (2010)

25. Zeleny, M.: An essay into a philosophy of MCDM: A way of thinking or another algorithm. Comput Oper Res 7(19), 563-566 (1992)

26. Roy, B.: Methodologie Multicritere D Aide a la Decision Economica, pp. 179-201. University of South Carolina Press, ??? (1985)

27. Guitouni, A., Martel, J.-M.: Tentative guidelines to help choosing an appropriate MCDA method. European Journal of Operational Research 109(2), 501-521 (1998). doi:10.1016/S0377-2217(98)00073-3

28. Almeida, A.T., Almeida, J.A., Costa, A.P.C.S., De Almeida-Filho, A.T.: A new method for elicitation of criteria weights in additive models: Flexible and interactive tradeoff. European Journal of Operational Research 250(1), 179-191 (2016). doi:10.1016/j.ejor.2015.08.058

29. Keeney, R.L., Raiffa, H.: Decisions with Multiple Objectives. New York: John Wiley and Sons (1976)

30. Gusmão, A.P.H., Medeiros, C.P.: A Model for Selecting a Strategic Information System Using the FITradeoff. Hindawi Publishing (2016)

31. Hongoh, V., Michel, P., Gosselin, P., Samoura, K., Ravel, A., Campagna, C., Cissá, H.D., Waaub, J.P. Multi-stakeholder decision aid for improved prioritization of the public health impact of climate sensitive infectious diseases. International Journal of Environmental Research and Public Health 13(4) (2016). doi:10.3390/ijerph13040419

32. LINS, A.N.L.: DECISÕES EM MEDICINA: UMA ABORDAGEM POR TEORIA DA DECISÃO. PhD thesis, UNIVERSIDADE FEDERAL DE PERNAMBUCO PROGRAMA (2009)

33. Brasil, M.d.S.: Política Nacional de Atenção às Urgências, pp. 1-138 (2006) doi:10.1017/CBO9781107415324.004. arXiv:1011.1669v3

34. Reis, F.M., Martins, D.M., Arruda, A.D., Linhares, A.O.M.: Manual De Atendimento Pré-hospitalar. EDITORA SANAR, ??? (2018). https://books.google.com.br/books?id=RKQJwwEACAAJ

35. Johnson, M., O'Hara, R., Hirst, E., Weyman, A., Turner, J., Mason, S., Quinn, T., Shewan, J., Siriwardena, A.N.: Multiple triangulation and collaborative research using qualitative methods to explore decision making in pre-hospital emergency care. BMC Medical Research Methodology 17(1), 11 (2017). doi:10.1186/s12874-017-0290-z

36. Hammersley, M., Atkinson, P.: Ethnography Principles in Practice. Routledge, Ney York: Routledge (2019)

37. Brasil, M.d.S.: Protocolos de Suporte Avançado de Vida, (2016)

38. Brasil, M.d.S.: Protocolos de Suporte Básico de Vida, (2016)

39. EDWARDS, W., BARRON, F.H.: SMARTS and SMARTER: Improved simple methods for multiattribute utility measurement. Organizational behavior and human decision processes 60(3), 306-325 (1994)

Figures

Figure 1 The Operation of SAMU/192

Figure 2 Decision criteria assessed by the expert group

Figure 3 Objective, criteria and alternatives of the proposed model 
Figure 4 Weight ranges

Figure 5 Alternatives found during the execution of the sensitivity analysis

Table 1 Complementary data associated with the victims.

\begin{tabular}{|c|l|l|l|l|}
\hline Information & Victim 1 & Victim 2 & Victim 3 & Victim 4 \\
\hline \multirow{2}{*}{ Location } & $\begin{array}{l}\text { Pajuçara - } \\
12,4 \mathrm{~km}-30 \mathrm{~m}\end{array}$ & $\begin{array}{l}\text { Lagoa Azul - } \\
12,1 \mathrm{~km}-27 \mathrm{~m}\end{array}$ & $\begin{array}{l}\text { Nossa Senhora } \\
\text { da Apresentação - } \\
11,8 \mathrm{Km} 28 \mathrm{~m}\end{array}$ & $\begin{array}{l}\text { Ponta Negra Village - } \\
12,0-30 \mathrm{~m}\end{array}$ \\
\hline \multirow{4}{*}{$\begin{array}{l}\text { Location's } \\
\text { Characteristcs }\end{array}$} & $\begin{array}{l}\text { Traffic congestion; } \\
\text { native vegetation area; } \\
\text { no paving; } \\
\text { raining; } \\
\text { there is insistence } \\
\text { by people close } \\
\text { to the victim }\end{array}$ & $\begin{array}{l}\text { Traffic congestion; } \\
\text { duplicate road; } \\
\text { on the road; } \\
\text { high incidence } \\
\text { of sunlight; } \\
\text { agglomeration } \\
\text { of people }\end{array}$ & $\begin{array}{l}\text { Traffic congestion; } \\
\text { narrow streets; } \\
\text { Victim is } \\
\text { on the road } \\
\text { exposed to } \\
\text { change of climate }\end{array}$ & $\begin{array}{l}\text { Heavy traffic; } \\
\text { duplicate road; } \\
\text { victim is home; } \\
\text { there is insistence } \\
\text { by relatives }\end{array}$ \\
\hline
\end{tabular}

Table 2 Final list of criteria related to the victim's health.

\begin{tabular}{|c|c|c|c|}
\hline Dimension & Criteria & Description & Scale \\
\hline \multirow{6}{*}{$\begin{array}{l}\text { Criteria } \\
\text { related to } \\
\text { the victim's } \\
\text { health }\end{array}$} & $\begin{array}{l}\text { Airways and } \\
\text { oxygenation }\end{array}$ & $\begin{array}{c}\text { The victim breathes normally, } \\
\text { does not breathe or has a loud or } \\
\text { altered breathing. } \\
\text { This has a direct impact } \\
\text { on the victim's health. }\end{array}$ & \multirow{6}{*}{$\begin{array}{c}\text { They're on a verbal } \\
\text { scale, ranging from } \\
\text { one to four. } \\
\text { Where } 1 \text { stands for Harmless } \\
\text { and } 4 \text { Severe. A higher } \\
\text { value is preferable to } \\
\text { a lower value. (Maximization). }\end{array}$} \\
\hline & State of consciousness & $\begin{array}{c}\text { The victim seems normal, } \\
\text { confused, unconscious, } \\
\text { had a seizure or loosened sphincters. }\end{array}$ & \\
\hline & Pulse & $\begin{array}{c}\text { Investigation of the victim's } \\
\text { pulse, the doctor wants } \\
\text { to investigate if it's normal, } \\
\text { fast, slow or non existent. }\end{array}$ & \\
\hline & $\begin{array}{l}\text { Airways and } \\
\text { oxygenation }\end{array}$ & $\begin{array}{c}\text { The victim breathes normally, } \\
\text { does not breathe or has a loud or } \\
\text { altered breathing. } \\
\text { This has a direct impact on the victim's health. }\end{array}$ & \\
\hline & Trauma & $\begin{array}{l}\text { The doctor wants to know } \\
\text { if there's visible bleeding, } \\
\text { body deformity, burns, } \\
\text { incarceration or confinement }\end{array}$ & \\
\hline & $\begin{array}{l}\text { Victim's } \\
\text { health history }\end{array}$ & $\begin{array}{l}\text { Historical data of the ailment, } \\
\text { recurrence, hereditary diseases, } \\
\text { or any other information that } \\
\text { might contribute to the treatment. }\end{array}$ & \\
\hline
\end{tabular}

List of Abbreviations

MCDA - Multicriteria Decision Analysis.

EMS - Emergency Medical Services.

Samu/192 - Mobile Emergency Care Service.

DM - Decision Maker.

FITradeoff - Flexible and Interactive Tradeoff method.

DSS - Decision Support System.

RN - Rio Grande do Norte.

RPs - Regulating Physicians.

TARMs - Medical Regulation Technicians.

RO - Radio Operator.

USA - Advanced Support Unit.

Additional Files

Additional file 1 - Sample additional file title

Additional file descriptions text (including details of how to view the file, if it is in a non-standard format or the file

extension). This might refer to a multi-page table or a figure.

Additional file 2 - Sample additional file title

Additional file descriptions text. 
Table 3 Final list of criteria related to the victim and external factors.

\begin{tabular}{|c|c|c|c|}
\hline Dimension & Criteria & Description & Scale \\
\hline \multirow{3}{*}{$\begin{array}{l}\text { Criteria } \\
\text { related } \\
\text { to the victim }\end{array}$} & Age & Victim's Age. & $\begin{array}{l}\text { It's represented by the age of } \\
\text { the victim. A lower value is } \\
\text { preferable to a higher } \\
\text { value. (Minimization) }\end{array}$ \\
\hline & Location & $\begin{array}{l}\text { The location of } \\
\text { the victim, the distance } \\
\text { from the relief facility. }\end{array}$ & $\begin{array}{c}\text { It's represented by the distance } \\
\text { from the victim to the ambulance, } \\
\text { expressed in } \mathrm{Km} \text {. A smaller } \\
\text { value is preferable to } \\
\text { a higher value. (Minimization) }\end{array}$ \\
\hline & $\begin{array}{l}\text { Access to } \\
\text { healthcare }\end{array}$ & $\begin{array}{l}\text { If the victim has a health } \\
\text { plan, if the victim has } \\
\text { and is able to be removed } \\
\text { to a medical facility } \\
\text { by their own means. }\end{array}$ & \multirow{2}{*}{$\begin{array}{l}\text { The measurement is a verbal scale, } \\
\text { ranging from } 1 \text { to } 4 \text {. Where } 1 \\
\text { means very bad and } 4 \text { means very good } \\
\text { (very bad, bad, good, very good). A lower } \\
\text { value is preferable to a } \\
\text { higher value. (Minimization) }\end{array}$} \\
\hline \multirow[t]{2}{*}{$\begin{array}{l}\text { Criteria } \\
\text { related } \\
\text { to external } \\
\text { factors }\end{array}$} & $\begin{array}{c}\text { Access to } \\
\text { victim's location }\end{array}$ & $\begin{array}{l}\text { If the address is in } \\
\text { rural areas, unpaved, } \\
\text { areas with greater presence } \\
\text { of vegetation, or regions } \\
\text { with high crime rate. } \\
\text { If there's slow traffic } \\
\text { or congestion. }\end{array}$ & \\
\hline & $\begin{array}{c}\text { Social } \\
\text { commotion }\end{array}$ & $\begin{array}{l}\text { It would be the discontent } \\
\text { or outrage resulting from } \\
\text { the victim's situation. } \\
\text { If the people close to } \\
\text { the victim who are } \\
\text { awaiting service insistently } \\
\text { return the call to } \\
\text { the regulatory center, } \\
\text { requesting immediate service. } \\
\text { If the victim is exposed } \\
\text { to any weather conditions } \\
\text { that are more intense. }\end{array}$ & $\begin{array}{c}\text { They are on a verbal scale, } \\
\text { ranging from } 1 \text { to } 4 \text {. Where } 1 \\
\text { means none and } 4 \text { means high } \\
\text { (none, low, medium, high). } \\
\text { A higher value is preferable } \\
\text { to a lower value. } \\
\text { (Maximization). }\end{array}$ \\
\hline
\end{tabular}

Table 4 Decision Matrix

\begin{tabular}{|c|c|c|c|c|c|c|c|c|c|c|}
\hline \multirow{3}{*}{ Alternatives } & \multicolumn{10}{|c|}{ Criteria } \\
\hline & \multicolumn{3}{|c|}{ Victim } & \multicolumn{5}{|c|}{ Victim's health status } & \multicolumn{2}{|c|}{ External factors } \\
\hline & $\begin{array}{l}\text { Location } \\
\text { KM }\end{array}$ & Age & $\begin{array}{l}\text { Access to } \\
\text { healthcare }\end{array}$ & $\begin{array}{c}\text { Airways } \\
\text { and } \\
\text { oxygenation }\end{array}$ & $\begin{array}{l}\text { State of } \\
\text { consciousness }\end{array}$ & Pulse & Trauma & $\begin{array}{c}\text { Victim's } \\
\text { health history }\end{array}$ & $\begin{array}{l}\text { Access to } \\
\text { victim's } \\
\text { location }\end{array}$ & $\begin{array}{c}\text { Social } \\
\text { commotion }\end{array}$ \\
\hline Victim 1 & 12,4 & 22 & 2 & 4 & 1 & 1 & 1 & 4 & 1 & 3 \\
\hline Victim 2 & 12,1 & 40 & 1 & 4 & 4 & 4 & 4 & 1 & 3 & 3 \\
\hline Victim 3 & 11,8 & 23 & 3 & 3 & 4 & 3 & 4 & 1 & 3 & 2 \\
\hline Victim 4 & 12 & 60 & 2 & 3 & 4 & 2 & 1 & 4 & 2 & 2 \\
\hline
\end{tabular}


Figures

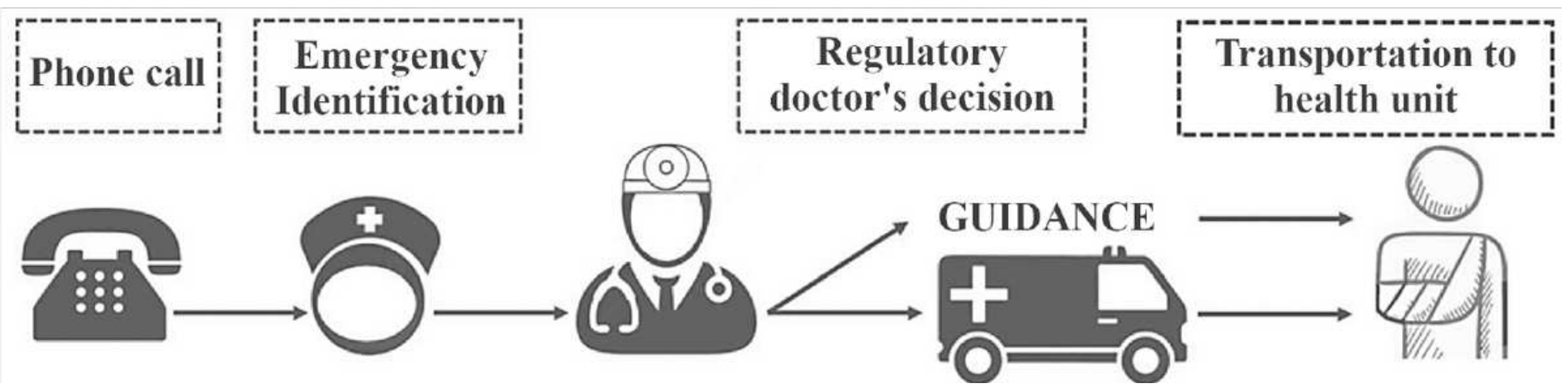

Figure 1

The Operation of SAMU/192

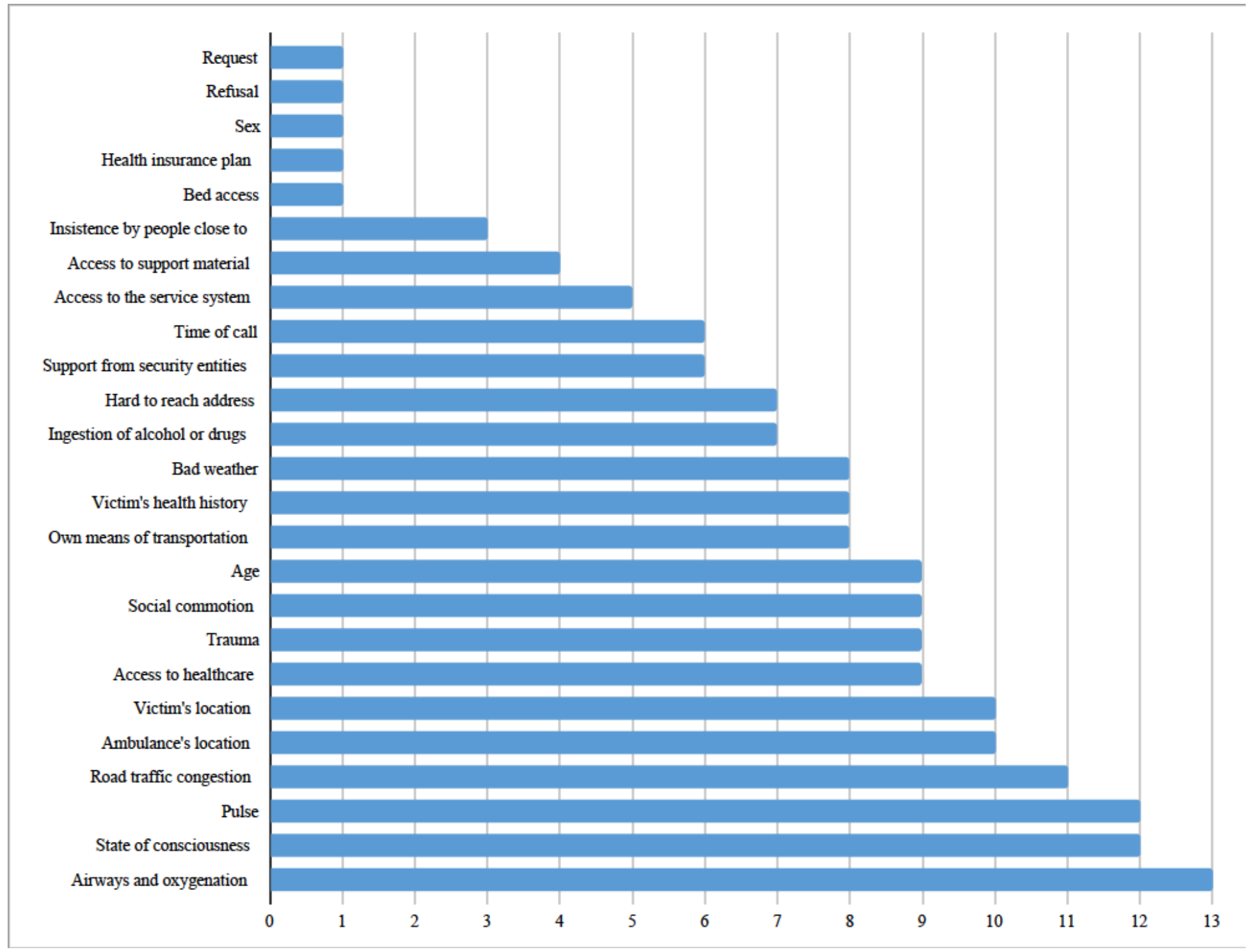

Figure 2 
Decision criteria assessed by the expert group

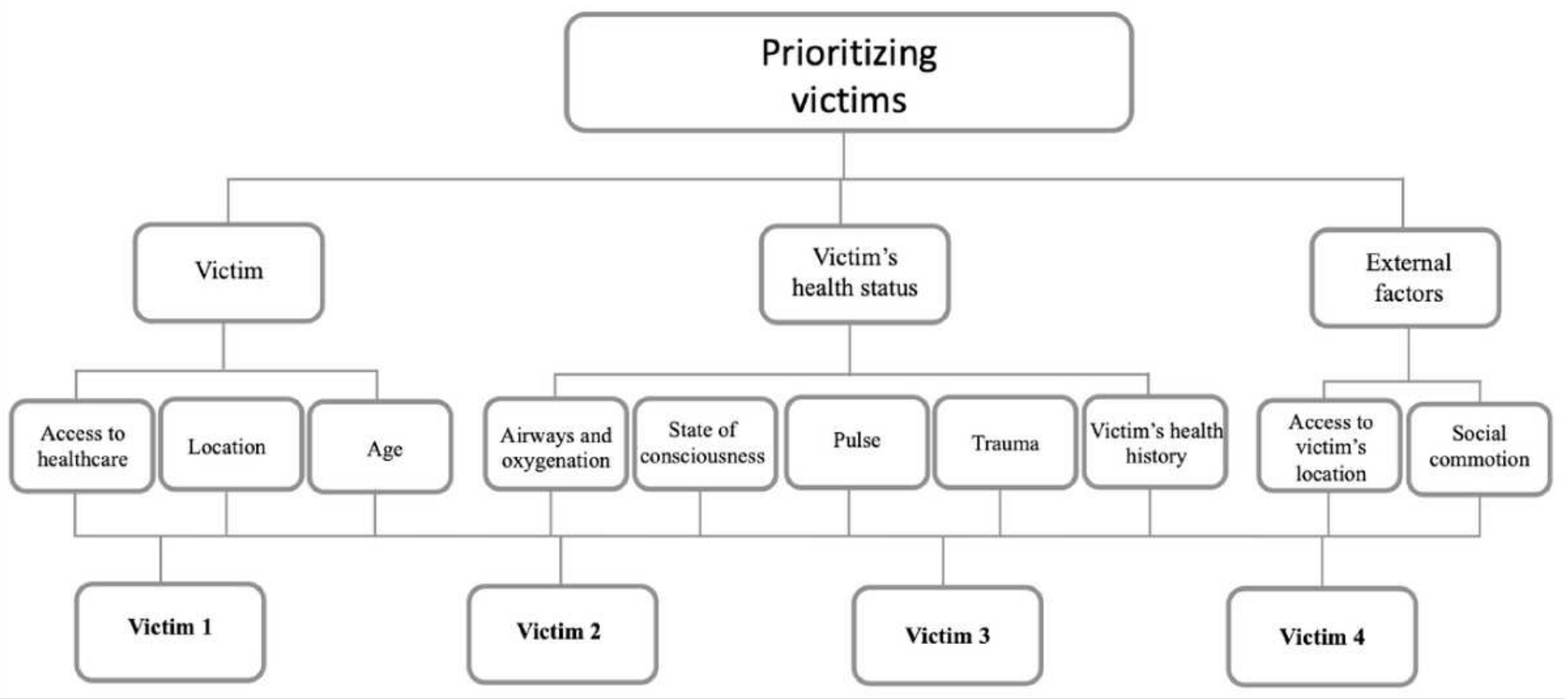

\section{Figure 3}

Objective, criteria and alternatives of the proposed model
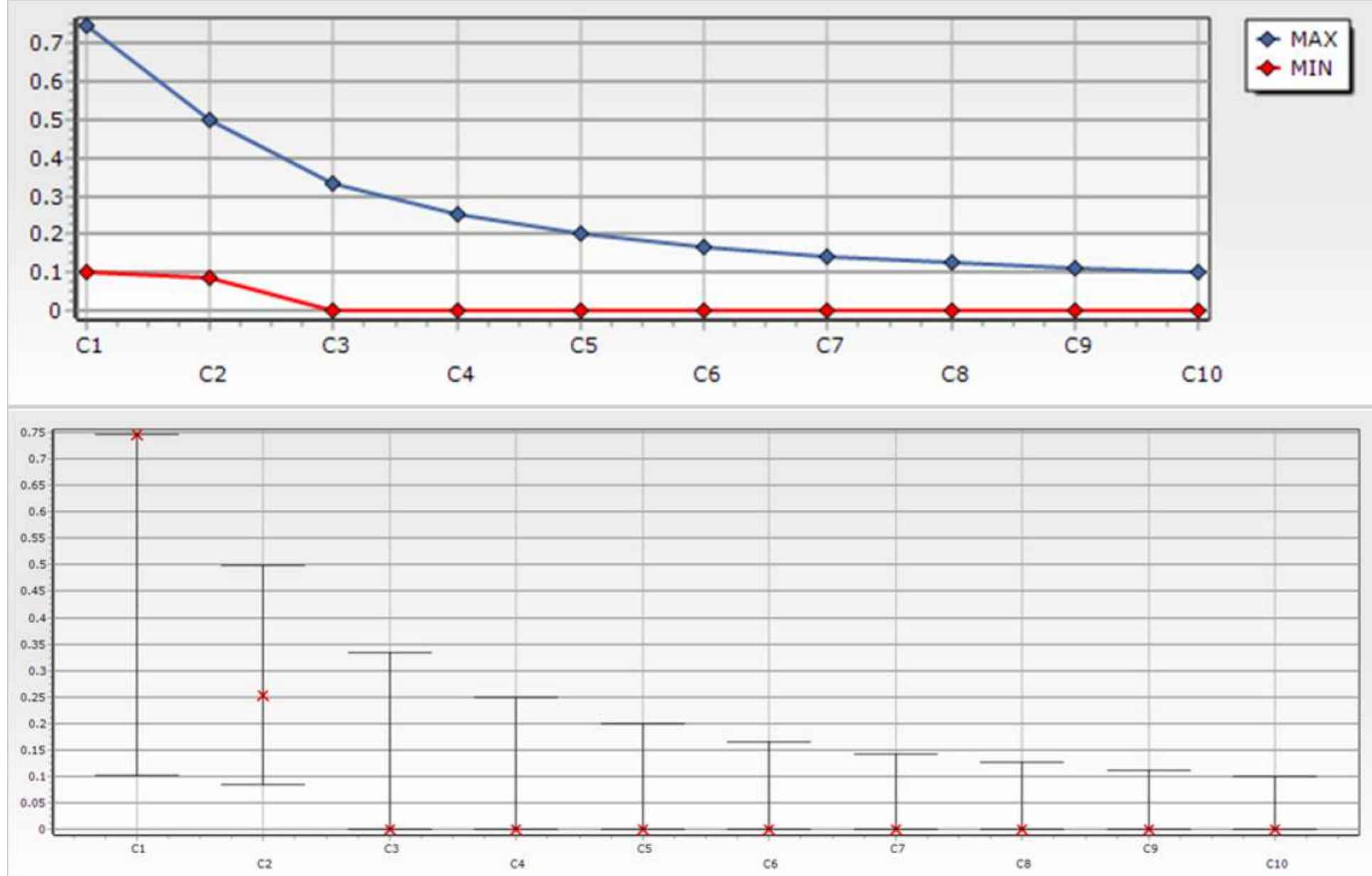
Figure 4

Weight ranges

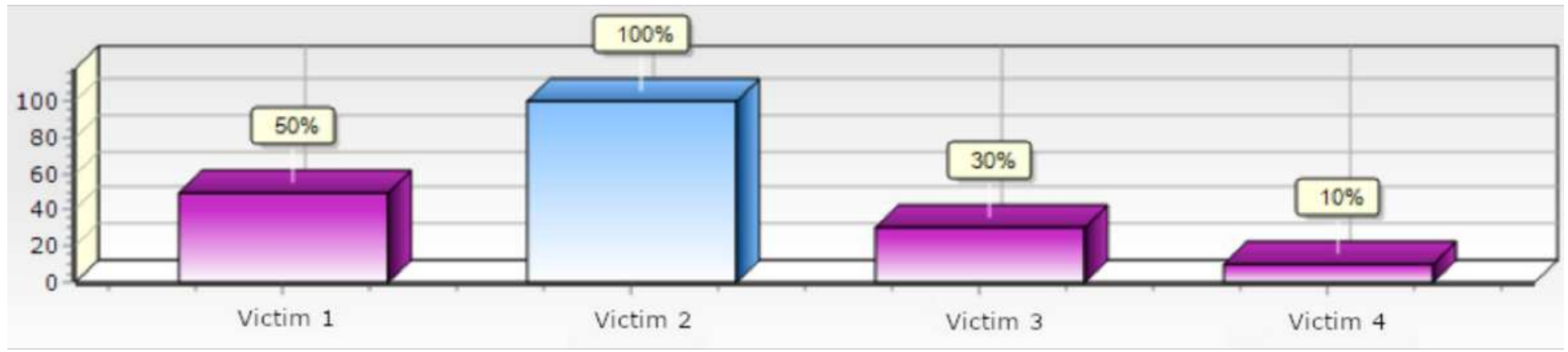

Figure 5

Alternatives found during the execution of the sensitivity analysis 